\title{
Ivermectin for COVID-19: A living systematic review protocolo
}

AUTHORS:

\section{Luis Ortiz-Muñoz}

ORCID: 0000-0001-6449-2153

UC Evidence Center, Cochrane Chile Associated Center, Pontificia Universidad Católica de Chile, Santiago, Chile

\section{Francisca Verdugo-Paiva}

0000-0003-0199-9744

Fundación Epistemonikos, Santiago, Chile

UC Evidence Center, Cochrane Chile Associated Center, Pontificia Universidad Católica de Chile, Santiago, Chile

\section{Rocío Bravo-Jeria}

0000-0002-3744-5578

UC Evidence Center, Cochrane Chile Associated Center, Pontificia Universidad Católica de Chile, Santiago, Chile

\section{Macarena Morel-Marambio}

0000-0003-3410-3131

UC Evidence Center, Cochrane Chile Associated Center, Pontificia Universidad Católica de Chile, Santiago, Chile

\section{María Paz Acuña}

ORCID: 0000-0001-7003-495X

Unidad de Infectología, Hospital Dr Sótero del Río, Santiago, Chile

Unidad de Infectología, Hospital Clínico Dra Eloísa Díaz, La Florida, Santiago, Chile

\section{Gabriel Rada}

ORCID: 0000-0003-2435-0710

Epistemonikos Foundation, Santiago, Chile

UC Evidence Center, Cochrane Chile Associated Center, Pontificia Universidad Católica de Chile, Santiago, Chile

Internal Medicine Department, Faculty of Medicine, Pontificia Universidad Católica de Chile, Santiago, Chile

\section{COVID-19 L-OVE Working Group}

\section{Corresponding author:}

Luis Ortiz-Muñoz

Email address: leortizmunoz@uc.cl

Postal address: Diagonal Paraguay \#476, Santiago, Chile 


\section{ABSTRACT}

\section{Objective}

This living systematic review aims to provide a timely, rigorous and continuously updated summary of the evidence available on the role of ivermectine in the treatment of patients with COVID-19 .

\section{Data sources}

We will conduct searches in the special L.OVE (Living OVerview of Evidence) platform for COVID-19, a system that maps PICO questions to a repository, maintained through regular searches in PubMed/Medline, Embase, Cochrane Central Register of Controlled Trials (CENTRAL) and other 33 trial registries, preprint servers, repositories and websites relevant to COVID-19. All the searches will cover the day before submission. No date or language restrictions will be applied.

\section{Eligibility criteria for selecting studies and methods}

We adapted an already published common protocol for multiple parallel systematic reviews to the specificities of this question.

We will include randomised trials evaluating the effect of ivermectine, as monotherapy or in combination with other drugs, versus placebo or no treatment in patients with COVID-19. Randomised trials evaluating ivermectine in infections caused by other coronaviruses, such as MERS-CoV and SARS-CoV, and non-randomised studies in COVID-19 will be searched in case no direct evidence from randomised trials is found, or if the direct evidence provides low- or very low-certainty for critical outcomes.

Two reviewers will independently screen each study for eligibility, extract data, and assess the risk of bias. We will pool the results using meta-analysis and will apply the GRADE system to assess the certainty of the evidence for each outcome.

A living, web-based version of this review will be openly available during the COVID-19 pandemic. We will resubmit it every time the conclusions change or whenever there are substantial updates.

\section{Ethics and dissemination}

No ethics approval is considered necessary. The results of this review will be widely disseminated via peer-reviewed publications, social networks and traditional media.

\section{PROSPERO Registration}

CRD42020189554

\section{Keywords}

COVID-19, severe acute respiratory syndrome coronavirus 2, Coronavirus Infections, Systematic review, Ivermectin, Antiparasitic Agents 


\section{INTRODUCTION}

COVID-19 is an infection caused by the SARS-CoV-2 coronavirus [1]. It was first identified in Wuhan, China, on December 31, 2019 [2]; five months later, more than six million cases of contagion had been identified across 188 countries [3]. On March 11, 2020, WHO characterised the COVID-19 outbreak as a pandemic [1].

While the majority of cases result in mild symptoms, some might progress to pneumonia, acute respiratory distress syndrome and death $[4],[5],[6]$. The case fatality rate reported across countries, settings and age groups is highly variable, but it ranges from about $0.5 \%$ to $10 \%$ [7]. In hospitalised patients it has been reported to be higher than $10 \%$ in some centres [8].

The Ivermectin is an antiparasitic that in human medicine is used in the form of tablets for the treatment of some inflammatory processes caused by parasites and in the form of a cream for the treatment of rosacea.

In vitro studies demonstrated an antiviral activity of ivermectin against SARS-CoV [9] However, there is none publishments about the results in clinical studies done in humans[10]. However, several systematic reviews and comprehensive syntheses evaluating the role of ivermectin concluded that its efficacy could not be established. [11], [12], [13].

Using innovative and agile processes, taking advantage of technological tools, and resorting to the collective effort of several research groups, this living systematic review aims to provide a timely, rigorous and continuously updated summary of the evidence available on the effects of ivermectine in patients with COVID-19.

\section{METHODS}

This manuscript complies with the 'Preferred Reporting Items for Systematic reviews and Meta-Analyses' (PRISMA) guidelines for reporting systematic reviews and meta-analyses [14].

A protocol stating the shared objectives and methodology of multiple evidence syntheses (systematic reviews and overviews of systematic reviews) to be conducted in parallel for different questions relevant to COVID-19 was published elsewhere [15]. The review was registered in PROSPERO with the number CRD42020189554 and a detailed protocol was uploaded to a preprint server [16] 


\section{Search strategies}

\section{Electronic searches}

Our literature search was devised by the team maintaining the L.OVE platform (https://app.iloveevidence.com), using the following approach:

Identification of terms relevant to the population and intervention components of the search strategy, using Word2vec technology [17] to the corpus of documents available in Epistemonikos Database.

Discussion of terms with content and methods experts to identify relevant, irrelevant and missing terms.

Creation of a sensitive boolean strategy encompassing all the relevant terms

Iterative analysis of articles missed by the boolean strategy, and refinement of the strategy accordingly.

Our main search source will be a repository developed and maintained by Epistemonikos Foundation through the screening of different sources relevant to COVID-19. At the time of submitting this protocol, this repository includes more than 2,6 million articles, with 41357 directly relevant to Coronavirus disease, coming from the following databases, trial registries, preprint servers, repositories and websites relevant to COVID-19: Epistemonikos database, Pubmed, EMBASE, CENTRAL, ICTRP Search Portal, Clinicaltrials.gov, ISRCTN registry, Chinese Clinical Trial Registry, Iranian Registry of Clinical Trials, EU Clinical Trials Register: Clinical trials for covid-19, NIPH Clinical Trials Search (Japan), Clinical Research Information System (Korea), medRxiv Preprints, bioRxiv Preprints, Microsoft Academic, WHO COVID-19 database, Google Scholar, Cochrane COVID-19 Study Register, NIHR: Innovation observatory: COVID-19 Updates, COVID-19: a living systematic map of the evidence, COVID-evidence website, Live map of COVID-19 evidence, LITCOVID: US National Library of Medicine, COVID-19 Special Collection (JBI)), Coronavirus disease (COVID-2019) R\&D, Coronavirus (COVID-19) Resource Hub, Coronavirus Research Repository (Elsevier Connect), Coronavirus (COVID-19) (CDC), Orientación sobre la COVID-19 y últimas investigaciones en las Américas (PAHO), COVID-19. Información confiable para la toma de decisiones, New England Journal of Medicine: Coronavirus (Covid-19), BMJ: Coronavirus (covid-19): Latest news and resources, Lancet: COVID-19 Resource Centre, JAMA: Coronavirus Disease 2019 (COVID-19), Oxford COVID-19 Evidence Service, NICE Rapid Guideline and Summaries on COVID-19, Coronavirus disease (COVID-19) - European Medicines Agency (EMA), Coronavirus Disease 2019 (COVID-19) - U.S. Food \& Drug Administration (FDA), SSRN Preprints, Research Square Preprints, arXiv Preprints.

The last version of the methods, the total number of sources screened, and a living flow diagram and report of the project is updated regularly on our website [18].

The searches will cover from the inception date of each database until the day before submission. No study design, publication status or language restriction will be applied. 
The following strategy is used to identify in the repository the articles potentially relevant for Coronavirus infection (COVID-19 and other coronavirus infections affecting humans):

(coronavir* OR coronovirus* OR betacoronavir* OR "beta-coronavirus" OR "beta-coronaviruses" OR "corona virus" OR "virus corona" OR "corono virus" OR "virus corono" OR hcov* OR "covid-19" OR covid19* OR "covid 19" OR "2019-ncov" OR cv19* OR "cv-19" OR "cv 19" OR "n-cov" OR ncov* OR (wuhan* and (virus OR viruses OR viral)) OR sars* OR sari OR (covid* and (virus OR viruses OR viral)) OR "severe acute respiratory syndrome" OR mers* OR "middle east respiratory syndrome" OR "middle-east respiratory syndrome" OR "covid-19-related" OR "2019-ncov-related" OR "cv-19-related" OR "n-cov-related") AND (ivermectin* OR stromectol* OR soolantra*)

\section{Other sources}

In order to identify articles that might have been missed in the electronic searches, we will do the following:

- Screen the reference lists of other systematic reviews and evaluate in full text all the articles they include.

- Scan the reference lists of selected guidelines, narrative reviews and other documents.

- Conduct cross-citation search in Google Scholar and Microsoft Academic, using each included study as the index reference.

- Review the reference list of each included study.

\section{Eligibility criteria}

Types of studies

This living review will preferentially include randomised trials. Non-randomised comparative studies will be included, but their information will be only used if no direct evidence from randomised trials if obtained, or if the certainty of the evidence for the critical outcomes resulting from the randomised trials is graded as low-or very low [19].

We will exclude studies evaluating the effects on animal models or in vitro conditions.

\section{Types of participants}

We will include trials assessing participants with COVID-19, as defined by the authors of the trials. Whenever we find substantial clinical heterogeneity on how the condition was defined, we will explore it using a sensitivity analysis.

In case we find no direct evidence from randomised trials, or if the evidence from randomised trials provides low- or very low-certainty evidence for critical outcomes, we will include information from randomised trials evaluating ivermectine in other coronavirus infections, such as MERS-CoV or SARS-CoV infections [19]. 
Type of interventions

The intervention of interest is. We will not restrict our criteria to any dosage, duration, timing or route of administration.

The comparison of interest will be placebo (ivermectine plus optimal treatment versus placebo plus optimal treatment) or no treatment (ivermectine plus optimal treatment versus optimal treatment).

Studies assessing ivermectine plus other drugs will be eligible if the cointerventions are identical in both intervention and comparison groups.

Studies evaluating ivermectine in combination with other active drugs versus placebo or no treatment will be also included.

\section{Type of outcomes}

We will not use the outcomes as an inclusion criteria during the selection process. Any article meeting all the criteria except for the outcome criterion will be preliminarily included and assessed in full text.

We used the core outcome set COS-COVID [20], the existing guidelines and reviews and the judgement of the authors of this review as an input for selecting the primary and secondary outcomes, as well as to decide upon inclusion. The review team will revise this list of outcomes, in order to incorporate ongoing efforts to define Core Outcomes Sets (e.g. COVID-19 Core Outcomes [21].

\section{Primary outcome}

- All-cause mortality

\section{Secondary outcomes}

- Invasive mechanical ventilation

- Extracorporeal membrane oxygenation

- Length of hospital stay

- Respiratory failure

- Serious adverse effects

- SARS-CoV negativity

\section{Other outcomes}

- Acute respiratory distress syndrome

- Total adverse effects

- Adverse effects leading to discontinuation

- Time to resolution of symptoms 


\section{Selection of studies}

The results of the literature search in the repository will be automatically incorporated into the L.OVE platform (automated retrieval), where the titles and abstracts will be independently screened by at least to reviewers against the inclusion criteria. We will obtain the full reports for all records that appear to meet the inclusion criteria or require further analysis to decide about their inclusion.

We will record the reasons for excluding trials in any stage of the search and outline the study selection process in a PRISMA flow diagram adapted for the purpose of this project .

\section{Extraction and management of data}

Using standardised forms, two reviewers will independently extract data from each included study. We will collect the following information: study design, setting, participant characteristics (including disease severity and age) and study eligibility criteria; details about the administered intervention and comparison, including dose and therapeutic scheme, duration, timing (i.e. time after diagnosis) and route of administration; the outcomes assessed and the time they were measured; the source of funding of the study and the conflicts of interest disclosed by the investigators; the risk of bias assessment for each individual study.

We will resolve disagreements by discussion, and one arbiter will adjudicate unresolved disagreements.

\section{Risk of bias assessment}

The risk of bias for each randomised trial will be assessed using the 'risk of bias' tool (RoB 2.0: a revised tool to assess risk of bias in randomised trials) [22]. We will consider the effect of assignment to the intervention for this review. Two reviewers will independently assess five domains of bias for each outcome result of all reported outcomes and time points. These five domains are: bias due to (1) the randomisation process, (2) deviations from intended interventions (effects of assignment to interventions at baseline), (3) missing outcome data, (4) measurement of the outcome, and (5) selection of reported results. Answers to signalling questions and collectively supporting information will lead to a domain-level judgement in the form of 'Low risk of bias', 'Some concerns', or 'High risk of bias'. These domain-level judgements will inform an overall 'risk of bias' judgement for each result. Discrepancies between review authors will be resolved by discussion to reach consensus. If necessary, a third review author will be consulted to achieve a decision.

We will assess the risks of bias of other study designs with the ROBINS-I tool (ROBINS-I: Risk Of Bias In Non-randomised Studies of Interventions) [23]. We will assess the following domains: bias due to confounding, bias in selection of participants into the study, bias in classification of interventions, bias due to deviations from intended interventions (effect of assignment to intervention), bias due to missing data, bias in measurement of outcomes and bias in the selection of the reported result. We will judge each domain as low risk, moderate risk, serious risk, critical risk, or no information, and evaluate individual bias items as described in ROBINS-I guidance. We will not consider time-varying confounding, as these confounders are not relevant in this setting [23]. 
We will consider the following factors as baseline potential confounders:

- Age

- Comorbidities (e.g. cardiovascular disease, renal disease, eye disease, liver disease)

- Co-interventions

- Severity, as defined by the authors (i.e respiratory failure vs respiratory distress syndrome vs ICU requirement).

\section{Measures of treatment effect}

For dichotomous outcomes, we will express the estimate of treatment effect of an intervention as risk ratios (RR) or odds ratios (OR) along with $95 \%$ confidence intervals (Cl).

For continuous outcomes, we will use mean difference and standard deviation (SD) to summarise the data using a $95 \% \mathrm{Cl}$. Whenever continuous outcomes are measured using different scales, the treatment effect will be expressed as a standardised mean difference (SMD) with $95 \% \mathrm{Cl}$. When possible, we will multiply the SMD by a standard deviation that is representative from the pooled studies, for example, the SD from a well-known scale used by several of the studies included in the analysis on which the result is based. In cases where the minimally important difference (MID) is known, we will also present continuous outcomes as MID units or inform the results as the difference in the proportion of patients achieving a minimal important effect between intervention and control [24].

Then, these results will be displayed on the 'Summary of Findings Table' as mean difference [24].

\section{Strategy for data synthesis}

If we include more than one trial we will conduct a formal quantitative synthesis (meta-analysis) for studies clinically homogeneous using RevMan 5 [25], using the inverse variance method with the random-effects model. For any outcomes where data were insufficient to calculate an effect estimate, a narrative synthesis will be presented, describing the direction and size of the effects, along with any reported measure of precision.

\section{Subgroup and sensitivity analysis}

We will perform subgroup analysis according to the definition of severe COVID-19 infection (i.e respiratory failure vs respiratory distress syndrome vs ICU requirement). In case we identify significant differences between subgroups (test for interaction $<0.05$ ) we will report the results of individual subgroups separately.

We will perform sensitivity analysis excluding high risk of bias studies, and if non-randomised studies are used, excluding studies that did not report adjusted estimates. In cases where the primary analysis effect estimates and the sensitivity analysis effect estimates significantly differ we will either present the low risk of bias (adjusted sensitivity analysis estimates) or present the primary analysis estimates but downgrading the certainty of the evidence because of risk of bias. 


\section{Assessment of certainty of evidence}

The certainty of the evidence for all outcomes will be judged using the Grading of Recommendations Assessment, Development and Evaluation working group methodology (GRADE Working Group)[25], across the domains of risk of bias, consistency, directness, precision and reporting bias. Certainty will be adjudicated as high, moderate, low or very low. For the main comparisons and outcomes, we will prepare Summary of Findings (SoF) tables [24], [25] and also interactive Summary of Findings tables (http://isof.epistemonikos.org/).

\section{Living evidence synthesis}

An artificial intelligence algorithm deployed in the Special L.OVE of Coronavirus (COVID-19)and will provide instant notification of articles with a high likelihood to be eligible. The authors will review them, will decide upon inclusion, and will update the living web version of the review accordingly. We will consider resubmission to a journal if there is a change in the direction of the effect on the critical outcomes or a substantial modification to the certainty of the evidence.

This review is part of a larger project set up to produce multiple parallel systematic reviews relevant to COVID-19 [15]. 


\section{NOTES}

\section{Acknowledgements}

The members of the COVID-19 L.OVE Working Group and Epistemonikos Foundation have made possible to build the systems and compile the information needed by this project. Epistemonikos is a collaborative effort, based on the ongoing volunteer work of over a thousand contributors since 2012.

\section{Roles and contributions}

All the review authors drafted and revised the protocol, conducted article screening and data collection, and drafted and revised the review.

The COVID-19 L.OVE Working Group was created by Epistemonikos and a number of expert teams in order to provide decision makers with the best evidence related to COVID-19. Up-to-date information about the group and its member organisations is available here: epistemonikos.cl/working-group

\section{Competing interests}

All authors declare no financial relationships with any organisation that might have a real or perceived interest in this work. There are no other relationships or activities that might have influenced the submitted work.

\section{Funding}

This project was not commissioned by any organisation and did not receive external funding.

Epistemonikos Foundation is providing training, support and tools at no cost for all the members of the COVID-19 L.OVE Working Group.

\section{PROSPERO registration}

CRD42020189554

\section{Ethics}

As researchers will not access information that could lead to the identification of an individual participant, obtaining ethical approval was waived.

\section{Data sharing}

All data related to the project will be available. Epistemonikos Foundation will grant access to data. 


\section{REFERENCES}

1. World Health Organization. Director-General's remarks at the media briefing on 2019-nCoV on 11 February 2020. [Internet] World Health Organization; 2020 [Accessed 2020 April 12] Available from:

https://www.who.int/dg/speeches/detail/who-director-general-s-remarks-at-the-media-briefing -on-2019-ncov-on-11-february-2020 .

2. Hui DS, I Azhar E, Madani TA, et al. The continuing 2019-nCoV epidemic threat of novel coronaviruses to global health - The latest 2019 novel coronavirus outbreak in Wuhan, China. Int J Infect Dis. 2020 Feb;91:264-266. Available from: doi:10.1016/j.ijid.2020.01.009

3. Dong E, Du H, Gardner L. An interactive web-based dashboard to track COVID-19 in real time. Lancet Infect Dis. 2020 Feb 19

4. Guan WJ, Ni ZY, Hu Y, et al. Clinical Characteristics of Coronavirus Disease 2019 in China. N Engl J Med 2020. Available from: doi:10.1056/NEJMoa2002032

5. Tavakoli A, Vahdat K, Keshavarz M. Novel Coronavirus Disease 2019 (COVID-19): An Emerging Infectious Disease in the 21st Century. BPUMS. 2020;22(6):432-450. Available from: doi:10.29252/ismj.22.6.432

6. Li LQ, Huang T, Wang YQ, Wang ZP, Liang Y, Huang TB, et al. 2019 novel coronavirus patients' clinical characteristics, discharge rate and fatality rate of meta-analysis. Journal of medical virology. 2020. Available from: doi:10.1002/jmv.25757

7. Global Covid-19 Case Fatality Rates [Internet] UK: Centre for Evidence-Based Medicine [Accessed 2020 April 12]. Available from:

https://www.cebm.net/covid-19/global-covid-19-case-fatality-rates/

8. Rodriguez-Morales AJ, Cardona-Ospina JA, Gutiérrez-Ocampo E, et al. Clinical, laboratory and imaging features of COVID-19: A systematic review and meta-analysis. Travel medicine and infectious disease. 2020;101623. Available from: doi:10.1016/j.tmaid.2020.101623

9. Caly L, Druce JD, Catton MG, Jans DA, Wagstaff KM. The FDAapproved drug ivermectin inhibits the replication of SARS-CoV-2 in vitro. Antiviral Res. 2020:104787. https://doi.org/10.1016/j.antiviral.2020.104787

10. Patrì, Angela, and Gabriella Fabbrocini. "Hydroxychloroquine and ivermectin: A synergistic combination for COVID-19 chemoprophylaxis and treatment?." Journal of the American Academy of Dermatology 82.6 (2020): e221.

11. Heidary, Fatemeh, and Reza Gharebaghi. "Ivermectin: a systematic review from antiviral effects to COVID-19 complementary regimen." The Journal of Antibiotics (2020): 1-10.

12. Hospital Alemão Oswaldo Cruz. Unidade de Avaliação de Tecnologias em Saúde, Hospital Sírio-Libanês. Núcleo de Avaliação de Tecnologias em Saúde. Ivermectin for COVID-19: rapid systematic review. 2020;[22fd1fe00027a412c0c23f8c6decc8e3e47f9358]

13. Perú. EsSalud. Instituto de Evaluación de Tecnologías en Salud e Investigación. Use of ivermectin for the treatment of patients with COVID-19. Perú. EsSalud. Instituto de Evaluación de Tecnologías 
en Salud e Investigación.. 2020; [b0d64f3c0686744affec209ebf0e20dc4f43bb45]

14. Moher D, Liberati A, Tetzlaff J, Altman DG; PRISMA Group. Preferred reporting items for systematic reviews and meta-analyses: the PRISMA statement. J Clin Epidemiol. 2009;62(10):1006-1012. doi:10.1016/j.jclinepi.2009.06.005

15. Rada G, Verdugo-Paiva F, Ávila C, Morel-Marambio M, Bravo-Jeria R, Pesce F, et al; COVID-19 L.OVE Working Group. Evidence synthesis relevant to COVID-19: a protocol for multiple systematic reviews and overviews of systematic reviews. Medwave 2020;20(3):e7867. Available from: doi:10.5867/medwave.2020.03.7867

16. Ortiz-Muñoz LE, Verdugo-Paiva F, Bravo-Jeria R, Morel M, Acuña MP, Rada G. Ivermectin for COVID-19: A living systematic review protocol 2020. doi:10.31219/osf.io/xsgke.

17. Github repository [Internet][Accessed 2020 April 3 Available from: https://github.com/dperezrada/keywords2vec

18. Methods for the special L.OVE of Coronavirus infection [Internet] Santiago: Epistemonikos Foundation [Accessed 2020 April 3]. Available from: https://app.iloveevidence.com/covid-19

19. Schünemann HJ, Cuello C, AkI EA, Mustafa RA, Meerpohl JJ, Thayer K, et al; GRADE Working Group. GRADE guidelines: 18. How ROBINS-I and other tools to assess risk of bias in nonrandomized studies should be used to rate the certainty of a body of evidence. J Clin Epidemiol. 2019 Jul;111:105-114. Available from: doi:10.1016/j.jclinepi.2018.01.012

20. Xinyao Jin, Bo Pang, Junhua Zhang, et al. Core Outcome Set for Clinical Trials on Coronavirus Disease 2019 (COS-COVID), Engineering, 2020. Available from: doi:10.1016/j.eng.2020.03.002

21. COVID-19 Core Outcomes [Internet]. [Accessed 2020 April 3]. Available from: https://www.covid-19-cos.org/

22. Sterne JAC, Savović J, Page MJ, Elbers RG, Blencowe NS, Boutron I, et al. RoB 2: a revised tool for assessing risk of bias in randomised trials. BMJ. 2019 Aug 28;366:14898. Available from: doi:10.1136/bmj.14898

23. Sterne JA, Hernán MA, Reeves BC, Savović J, Berkman ND, Viswanathan M, et al. ROBINS-I: a tool for assessing risk of bias in non-randomised studies of interventions. BMJ. 2016 Oct 12;355:i4919. Available from: doi:10.1136/bmj.i4919

24. Review Manager (RevMan) [Software]. Version 5.3.5 Copenhagen: The Nordic Cochrane Centre, The Cochrane Collaboration, 2014.

25. Guyatt GH, Oxman AD, Santesso N, et al. GRADE guidelines: 12. Preparing summary of findings tables-binary outcomes. J Clin Epidemiol [Internet] 2013 Feb [Accessed March 26] ;66(2):158-72. Available from: doi:10.1016/j.jclinepi.2012.01.012 